5. Compact Topological Groups. The following theorem is loosely related to Theorem 1 .

THEOREM 3. Let $G$ be any compact topological group whose manifold is homeomorphic with a subset of Cartesian n-space. Then any series of closed subgroups of $G$ can be well-ordered in the direction of increasing subgroups.

For the different group nuclei* are at most $(n+1)$ in number. And the index of the subgroup generated by any one of these nuclei in any larger closed subgroup having the same nucleus is finite.

But if we restrict ourselves to closed $T$-invariant subgroups, then the proof of Theorem 1 breaks down. For consider the additive group of residues modulo unity. The subgroups generated by $1 / 2,1 / 4,1 / 8, \cdots$ form one chief series, and those generated by $1 / 3,1 / 9,1 / 27, \cdots$ a second one, and yet the two have not a single factor-group in common.

Society of Fellows, Harvard University

\title{
LOCI OF $m$-SPACES JOINING CORRESPONDING POINTS OF $m+1$ PROJECTIVELY RELATED $n$-SPACES IN $r$-SPACE $\dagger$
}

BY B. C. WONG

Let $m+1 n$-spaces $S_{n}{ }^{(1)}, S_{n}{ }^{(2)}, \cdots, S_{n}{ }^{(m+1)}$ be given in general positions in an $r$-space $S_{r}$. It is convenient, but not necessary, to let $r=m n+m+n$. We shall assume that the given $n$-spaces are in an $S_{m n+m+n}$. Now suppose that these $n$-spaces are all projectively related, that is, to a given subspace in any one of them corresponds a definite subspace of the same number of dimensions in each of the others. These corresponding subspaces are themselves projectively related.

Now consider a group of corresponding points, one in each of the $m+1$ given $n$-spaces. These points determine an $m$-space.

* A group nucleus is a neighborhood of the identity; two group nuclei are considered the same if sufficiently small common neighborhoods of the origin are isomorphic.

† Presented to the Society, June 20, 1934. 
The given spaces being $n$-dimensional, we have $\infty^{n} m$-spaces so determined, forming an $(m+n)$-dimensional variety $V_{m+n}{ }^{N_{m, n}}$ of order $N_{m, n}$. In this note we are concerned with this variety $V_{m+n}{ }^{N_{m, n}}$. We shall determine, by elementary methods, its order and then write its equations. After stating a few of its properties, we shall, finally, describe the surfaces in which it is met by linear spaces of the proper number of dimensions.

To determine $N_{m, n}$, pass a hyperplane of $S_{m n+m+n}$ through $m$ of the given $n$-spaces, say $S_{n}{ }^{(1)}, S_{n}{ }^{(2)}, \cdots, S_{n}{ }^{(m)}$. This hyperplane intersects the remaining $n$-spaces $S_{n}^{(m+1)}$ in an $(n-1)$ space $S_{n-1}{ }^{(m+1)}$ to which correspond $m(n-1)$-spaces $S_{n-1}{ }^{(1)}$, $S_{n-1}{ }^{(2)}, \cdots, S_{n-1}{ }^{(m)}$ in $S_{n}{ }^{(1)}, S_{n}{ }^{(2)}, \cdots, S_{n}{ }^{(m)}$, respectively, and it intersects the variety $V_{m+n} N_{m, n}$ in a $V_{m+n-1}{ }^{N_{m, n}}$. This $V_{m+n-1} N_{m, n}$ is evidently composed of two varieties, $V_{m+n-1}{ }^{N_{m-1, n}}$ and $V_{m+n-1}{ }^{N, n-1}$, the former being the locus of the $\infty^{n}(m-1)$ spaces joining corresponding points of the $m$ projectively related $n$-spaces $S_{n}{ }^{(1)}, S_{n}{ }^{(2)}, \cdots, S_{n}{ }^{(m)}$ and the latter the locus of the $\infty^{n-1} m$-spaces joining corresponding points of the $m+1$ projectively related $(n-1)$-spaces $S_{n-1}^{(1)}, S_{n-1}^{(2)}, \cdots, S_{n-1}{ }^{(m+1)}$. Therefore, the order of $V_{m+n}{ }^{N_{m, n}}$ is

$$
N_{m, n}=N_{m-1, n}+N_{m, n-1} \text {. }
$$

To determine $N_{m-1, n}$ and $N_{m, n-1}$, we proceed in a similar manner and find that

$$
\begin{aligned}
& N_{m-1, n}=N_{m-2, n}+N_{m-1, n-1}, \\
& N_{m, n-1}=N_{m-1, n-1}+N_{m, n-2} .
\end{aligned}
$$

Continuing this process of derivation until it terminates, we find that we may write, after making the proper substitutions, either

or

$$
N_{m, n}=N_{m-1, n}+N_{m-1, n-1}+\cdots+N_{m-1,0}
$$

$$
N_{m, n}=N_{m, n-1}+N_{m-1, n-1}+\cdots+N_{0, n-1} \text {. }
$$

Either case yields, since $N_{x, 0}=N_{0, x}=1$, that is, the variety $V_{x}^{N_{x, 0}}$ or $V_{x}^{N_{0, x}}$ is just a linear $x$-space, the same result

$$
N_{m, n}=\left(\begin{array}{c}
m+n \\
m
\end{array}\right)=\left(\begin{array}{c}
m+n \\
n
\end{array}\right) \text {. }
$$

This is the order of the variety $V_{m+n}^{N_{m, n}}$. 
To derive the equations of the variety, it is convenient to take for the $m+1$ given $n$-spaces any group of $m+1$ non-intersecting $n$-spaces of the coordinate simplex of $S_{m n+m+n}$. We shall take the group of $n$-spaces whose equations are

$$
\begin{aligned}
& S_{n}^{(1)} \quad x_{0}: x_{1}: \cdots: x_{n}=u_{0}: u_{1}: \cdots: u_{n} \text {, } \\
& x_{n+1}=x_{n+2}=\cdots=x_{m n+m+n}=0 \text {; } \\
& S_{n}^{(2)} \quad x_{0}=x_{1}=\cdots=x_{n}=0 \text {, } \\
& x_{n+1}: x_{n+2}: \cdots: x_{2 n+1}=u_{0}: u_{1}: \cdots: u_{n} \text {, } \\
& x_{2 n+2}: x_{2 n+3}: \cdots: x_{m n+m+n}=0 \text {; } \\
& S_{n}^{(m+1)} x_{0}=x_{1}=\cdots=x_{m n+m-1}=0 \text {, } \\
& x_{m n+m}: x_{m n+m+1}: \cdots: x_{m n+m+n}=u_{0}: u_{1}: \cdots: u_{n} .
\end{aligned}
$$

The coordinates of any point in the $m$-space joining corresponding points of these $m+1 n$-spaces are given by

$$
x_{i n+i+j}=t_{i} u_{j}, \quad(i=0,1,2, \cdots, m ; j=0,1,2, \cdots, n) .
$$

These are, then, the equations of our $V_{m+n}{ }^{N_{m, n}}$ if we regard the $t$ 's and $u$ 's as variable parameters. Eliminating these parameters we obtain $\left(\begin{array}{c}m+1 \\ 2\end{array}\right)\left(\begin{array}{c}n+1 \\ 2\end{array}\right)$ quadratic equations given by the vanishing of all the two-rowed determinants of the matrix

$$
\left\|\begin{array}{cccc}
x_{0} & x_{1} & & x_{n} \\
x_{n+1} & x_{n+2} & \cdots & x_{2 n+1} \\
\cdot & \cdot & & \cdot \\
\cdot & \cdot & & \cdot \\
x_{m n+m} & x_{m n+m+1} & \cdots & x_{m n+m+n}
\end{array}\right\|
$$

We see that the variety $V_{m+n}{ }^{N}, n$ is the common intersection of $\left(\begin{array}{c}m+1 \\ 2\end{array}\right)\left(\begin{array}{c}n+1 \\ 2\end{array}\right)$ quadric hypersurfaces given by the above quadratic equations.

We have already defined our $V_{m+n}{ }^{N_{m, n}}$ as the locus of the $\infty^{n}$ $m$-spaces determined by corresponding points of $m+1$ projectively related $n$-spaces. If we interchange $m$ and $n$, the equations of the variety (and also the result for its order) remain unchanged and therefore we may define $V_{m+n}{ }^{N_{m, n}}$ as the locus of 
the $\infty^{m} n$-spaces joining corresponding points of $n+1$ projectively related $m$-spaces. Since the projectivity between $m+1$ given $n$-spaces in an $S_{m n+m+n}$ may be determined by the points of another given $n$-space, $S_{n}{ }^{(m+2)}$, that is, from a point of $S_{n}{ }^{(m+2)}$ one and only one $m$-space can be constructed incident with the $m+1$ given $n$-spaces, we see that $V_{m+n}{ }^{N} m, n$ is also the locus of the $\infty^{n} m$-spaces incident with $m+2$ given $n$-spaces. Similarly, it is the locus of the $\infty^{m} n$-spaces incident with $n+2$ given $m$-spaces.

From these definitions, we see that our variety contains a system of $\infty^{n} m$-spaces and a system of $\infty^{m} n$-spaces. No two $m$-spaces nor two $n$-spaces can intersect but each $m$-space meets each $n$-space in a point. Through each point of the variety pass one $m$-space and one $n$-space.

For $m=n=1$, we have a quadric surface in 3 -space. If $m=2$, $n=1$, we have a $V_{3}{ }^{3}$ in 5 -space which is the locus of the $\infty^{1}$ planes incident with 4 lines and at the same time the locus of the $\infty^{2}$ lines incident with 3 planes.

The case $m=1$ and $n$ general is of interest. The variety $V_{n+1}{ }^{n+1}$ in $S_{2 n+1}$ is the locus of the $\infty^{1} n$-spaces incident with $n+2$ lines and also the locus of the $\infty^{n}$ lines incident with $3 n$-spaces. Since $V_{n+1}^{n+1}$ is intersected by a general $n$-space of $S_{2 n+1}$ in $n+1$ points, we have the result that there are $n+1$ lines incident with $4 n$-spaces given in general positions in $S_{2 n+1}$. For $n=1$, we have the familiar case of the two transversals of 4 general lines in 3-space.

Now the variety $V_{m+n}^{N_{m, n}}$ is intersected by an $S_{m(n+1)}$ of $S_{m n+m+n}$ in a $V_{m}^{N_{m, n}}$ and by an $S_{n(m+1)}$ in a $V_{m}^{N_{m, n}}$. Both of these varieties are rational, the one representable upon an $S_{m}$ and the other representable upon an $S_{n}$, respectively. We may assume $m \geqq n$. Then, $V_{m}^{N_{m, n}}$ is the locus of $\infty^{n}(m-n)$-spaces and $V_{n}^{N_{m, n}}$ is the locus of $\infty^{n}$ points. Let us consider the case $n=2$. The variety $V_{m+2^{N}}{ }_{m, 2}$ or $V_{m+2}^{(m+1)(m+2) / 2}$ is now the locus of the $\infty^{2} m$-spaces joining corresponding points of $m+1$ given projectively related planes $\alpha, \beta, \gamma$ in an $S_{3 m+2}$, and is intersected by a given $S_{2 m+2}$ of $S_{3 m+2}$ in a rational surface $F$ of order $(m+1)(m+2) / 2$. Let the projectivity between these planes be determined by the points of a fourth plane, $\phi$. From a point $P$ of $\phi$ one and only one $m$-space $S_{m}$ can be drawn meeting $\alpha, \beta, \gamma$ each in a point. We shall take these points for corresponding points in the projectivity. Now $S_{m}$ meets the given $S_{2 m+2}$ in a point $P^{\prime}$ which is 
on $F$. Thus, we have a one-to-one correspondence between the points of $F$ and those of $\phi$.

It can be easily shown that there exist $m(m+1) / 2$ points $A_{i}$, $(i=1,2, \cdots, m(m+1) / 2)$, in the plane $\phi$ (or in any plane of $\left.S_{3 m+2}\right)$ from each of which an $m$-space can be constructed meeting $\alpha, \beta, \gamma$ each in a point and $S_{2 m+2}$ in a line. Therefore, $F$ has on it $m(m+1) / 2$ lines which are the images of $A_{\boldsymbol{i}}$ in $\phi$. If $P$ in $\phi$ describes a line not passing through any of the points $A_{i}$, the $m$-space $S_{m}$ describes a $V_{m+1}{ }^{m+1}$ in $S_{3 m+2}$ which is met by $S_{2 m+2}$ in a rational curve $C^{m+1}$ of order $m+1$ lying on $F$. The curve $C^{m+1}$ is the locus of the corresponding points $P^{\prime}$ and is the image of the line described by $P$. From what has just been said we can see easily that the fundamental curves of representation in $\phi$ are the $\infty^{2 m+2}$ curves of order $m+1$ all passing through the $m(m+1) / 2$ points $A_{i}$ whose images are the $(m+1)(m+2) / 2$-ic curves in which the $(2 m+1)$-spaces of $S_{2 m+2}$ intersect $F$.

A little calculation shows that the projection of the rational surface $F$ upon a 4 -space has $m(m-1)\left(m^{2}+7 m-6\right) / 8$ improper double points and that its projection upon a 3-space is of class $3 m^{2}$ and rank $2 m(m+1)$ and has a double curve whose order is $m(m+1)\left(m^{2}+5 m-2\right) / 8$, and upon which lie $2 m(2 m-1)$ pinch points. If we project $F$ from an $S_{2 m-2}$ containing $2 m-1$ general points of it upon a 3 -space, we have for projection a surface $F^{\prime}$ of order $\left(m^{2}-m+4\right) / 2$, class $3 m^{2}$, and rank $2\left(m^{2}-m+1\right)$. Its double curve is of order $m\left(m^{2}-1\right)(m-2) / 8$ and on it there are $2(2 m-1)(m-2)$ pinch points. The surface $F^{\prime}$ is representable upon a plane by the $\infty^{3}(m+1)$-ic curves all passing through $\left(m^{2}+5 m-2\right) / 2$ base points. All these results hold for $m \geqq 2$. For $m=2, F^{\prime}$ is a cubic surface whose representation upon a plane by means of the $\infty^{3}$ cubic curves through 6 given points is well known. If $m=3$, we have a quintic surface containing a nodal curve of order 3 with 10 pinch points. The fundamental curves of representation in this case are the $\infty^{3}$ quartic curves through 11 base points.

The University of California 